\title{
Atividade antioxidante de xantonas isoladas de espécies de Leiothrix (Eriocaulaceae)
}

\author{
Santos, L.C. ${ }^{1}$; Piacente, S. ${ }^{2}$; Montoro, P. ${ }^{2}$; Pizza, C. ${ }^{2}$; Vilegas, W. ${ }^{1}$ \\ ${ }^{1}$ Instituto de Química, Departamento de Química Orgânica; Universidade Estadual Paulista \\ Araraquara, SP; ${ }^{2}$ Departamento de Ciências Farmacêuticas, Universidade de \\ Estudos de Salerno, Fisciano, Itália.
}

Recebido para publicação em: 28/07/2003

Aceito para publicação em: 21/11/2003

\begin{abstract}
RESUMO: Leiothrix (Eriocaulaceae) é um gênero exclusivo da América do Sul, com 37 espécies restritas ao Brasil. Leiothrix flavescens (Bong.) Ruhland e Leiothrix curvifolia (Bong.) Ruhland, são espécies endêmicas em áreas dos Estados de Minas Gerais e Bahia. Existem na literatura poucos estudos químicos e biológicos realizados com espécies desse gênero. Neste trabalho, foi estudada a atividade antioxidante pelo ensaio TEAC (Trolox Equivalent Antioxidant Capacity) e de autoxidação, utilizando ácido linoléico, de xantonas isoladas dos extratos etanólicos de $L$. curvifolia e L. flavescens. As substâncias apresentaram moderada atividade antioxidante, quando comparadas com padrões de quercetina e de $\mathrm{BHT}$ (2, 6 - ditercbutil - 4 - metóxifenil).

Unitermos: Ação antioxidante; TEAC; Autoxidação; ácido linoléico; Eriocaulaceae; Leiotrhrix spp.; xantonas.

ABSTRACT: Antioxidant effects of xanthones isolated from Leiothrix species (Eriocaulaceae). Leiothrix (Eriocaulaceae) is an exclusively South American genus of which 37 species are restricted to Brazil. Leiothrix flavescens (Bong.) Ruhland and Leiothrix curvifolia (Bong.) Ruhland, are species endemic to areas of Minas Gerais and Bahia States. Chemical and biological investigations of this genus are scarse. The objective of this work was to investigate the antioxidant effect using TEAC and linoleic acid assays of the xanthones isolated from the ethanolic extract of these species. These substances showed a moderate antioxidant activity when compared to quercetin and to BHT.
\end{abstract}

Key Words: Antioxidant; TEAC; Linoleic acid; Eriocaulaceae; Leiothrix spp. Xanthones. 


\section{INTRODUÇÃO}

A geração de espécies reativas de oxigênio (EROs) é um processo normal na vida dos organismos vivos, mas elas são capazes de lesar componentes celulares, contribuindo, assim, para o desenvolvimento de várias doenças, como artrites inflamatórias (HADJIGOGOS, 2003), úlceras (LA CASA et al., 2000 ) e diabetes (EL-REMESSY et al., 2003), dentre outras. Em condições fisiológicas, essas espécies são removidas por sistemas antioxidantes, que podem ser vitaminas, proteínas ou enzimas (BANERJEE et al., 2003). Dentre os produtos naturais de origem vegetal com atividade antioxidante, os mais estudados são os flavonóides (RE et al, 1999).

O Brasil possui uma enorme diversidade biológica, especialmente com relação à sua flora. O interesse em realizar ensaios biológicos com substâncias isoladas de plantas da família Eriocaulaceae surgiu com o isolamento de um metabólito não-usual em plantas, mas freqüente em microorganismos dos gêneros Penicillium e Fusarium (HILL, 1986): a isocumarina paepalantina, isolada dos capítulos de Paepalanthus bromelioides, pertecentes à esta família (VILEGAS et al., 1990). Ensaios biológicos in vitro indicaram forte atividade antibiótica contra Bacillus cereus ( 7,5 $\mathrm{mg} / \mathrm{ml})$, Staphylococcus aureus $(7,5 \mathrm{mg} / \mathrm{ml})$, S. epidermidis $(15 \mathrm{mg} / \mathrm{ml})$ e Escherichia coli $(250$ $7,5 \mathrm{mg} / \mathrm{ml})$, quando comparada com a azacitidina $(0,102 \mathrm{mM})$, um fármaco utilizado como agente quimioterápico (VARANDA et al., 1997; TAVARES et al., 1999).

A família Eriocaulaceae possui ampla distribuição, sendo rica em espécies e com grande diversidade na América do Sul (GIULIETTI et al., 2000). Estas plantas sobrevivem em condições especiais de clima e solo, o que leva ao aparecimento de um grande número de espécies endêmicas, tornando os campos rupestres a formação vegetal com maior índice de endemismos no Brasil (JOLY, 1970). Elas são conhecidas popularmente como "sempre-vivas", por suas inflorescências de coloração paleácea e de grande durabilidade (GIULIETTI et al., 2000). Algumas espécies são largamente usadas para fins de decoração, sendo também produto de exportação do Brasil (DOKKEDAL, 2000).

Leiothrix é um gênero exclusivo da América do Sul, com 37 espécies restritas ao Brasil. Leiothrix flavescens (Bong.) Ruhland ocorre no Brasil, Venezuela e Peru. As outras espécies são endêmicas em áreas dos Estados de Minas Gerais e Bahia (GIULIETTI et al., 2000).

Uma classe de substâncias que não foi isolada de plantas dos gêneros (Paepalanthus e Syngonanthus), mas que foi encontrada no gênero Leiothrix (Eriocaulaceae) são as xantonas (SANTOS et al, 2001).

O estudo de xantonas é interessante não só pela investigação quimiossistemática, mas também devido à sua importância farmacológica (PERES; NAGEN, 1977). Vários trabalhos científicos já foram realizados evidenciando as importantes atividades farmacológicas apresentadas pelas xantonas. De todas, talvez a mais interessante seja a ação inibitória da enzima monoaminooxidase (MAO), relacionada com o tratamento de estados depressivos; as xantonas apresentaram um grande potencial de utilização por terem demonstrado atividade inibitória potente, seletiva e reversível desta enzima (USDIN, 1984; THULLA et al., 1993; GNERRE et al., 2001). Diversos trabalhos têm apresentado as xantonas como potenciais substâncias para o tratamento do câncer (LIN et al., 1996; ASANO et al. 1996; PACHUTA et al., 1986, LIOU et al., 1993; PERMANA et al., 1994). Algumas xantonas (naturais e sintéticas) apresentaram, ainda, ação antimicrobiana, inclusive contra Mycobacterium tuberculosis (GHOSAL; CHAUDHURI, 1975; GHOSAL et al., 1978), ação antifúngica (ROCHA et al., 1994), entre outras. Mais referências sobre tais potenciais atividades farmacológicas podem ser encontradas em Kuster e Rocha (2003). Por outro lado, a toxicidade das xantonas é ainda pouco estudada, e esse é um dos passos limitantes ao seu emprego terapêutico.

Rev. Bras. Farmacogn., V. 13, n. 2, jul.-dez. 2003. 
Neste trabalho, objetivou-se realizar a investigação da atividade antioxidante das xantonas $(1,2$ e 3$)$ isoladas dos extratos etanólicos dos capítulos de L. curvifolia e L. flavescens (Figura 1), através dos dois métodos abaixo descritos.

O ensaio TEAC (Trolox Equivalent Antioxidant Capacity) avalia espectrofotometricamente a habilidade relativa das substâncias antioxidantes em capturar o cátion radical 2,2'-azinobis(3etilbenzotiozolino-6-ácido sulfônico) (ABTS+ ${ }^{+}$, quando comparada com uma quantidade padrão do antioxidante sintético Trolox ${ }^{\circledR}$ (ácido 2-carboxílico-6-hidroxi-2,5,7,8-tetrametilcromano), um análogo da vitamina $E$, solúvel em água. A atividade dos compostos testados é expressa em valores TEAC, que é definido como a concentração de Trolox que possui a mesma atividade que $1 \mathrm{mM}$ da substância antioxidante investigada. Os compostos são considerados ativos quando o seu valor de TEAC é próximo ao da quercetina, flavonóide usado como substância de referência (RE et al., 1999).

No método de autoxidação, utilizando o ácido linoléico, os produtos da peroxidação desse são capazes de reagir com o $\beta$-caroteno, produzindo descoloração na solução de coloração amarelo-alaranjada. A presença de uma substância antioxidante na mistura protege o ácido linoléico da autoxidação, impedindo tal descoloração. A mistura reagente consiste em ácido linoléico, $\beta$ caroteno, água, a substância a ser investigada e o BHT (2,6-di-tercbutil-4-metóxifenol), que é usado como referência.

\section{MATERIALE MÉTODOS}

\section{Material vegetal}

Capítulos de L. curvifolia e L. flavescens foram coletados em fevereiro de 1998 e julho de 1984, respectivamente, em Dimantina, Minas Gerais. A identificação das espécies foi realizada pelo Prof. Dr. Paulo Takeo Sano do Instituto de Biociências da USP - SP. Um exemplar de cada espécie (exsicata) encontra-se depositado no herbário do IB-USP sob os números: L. curvifolia (WV-11, SANO, 861) e L. flavescens (CFCR 4680).

\section{Preparação do extrato e isolamento das xantonas}

Os capítulos de $L$. curvifolia (32g) e $L$. flavescens $(200 \mathrm{~g})$ foram secos em estufa a $40^{\circ} \mathrm{C} \mathrm{e}$, posteriormente, triados para separação dos capítulos e escapos. Em seguida, foram, separadamente, moídos e submetidos à maceração, ficando em contato com cada solvente orgânico por uma semana, (hexano, diclorometano, etanol). Após cada extração, o solvente foi concentrado em evaporador rotatório.

Parte do extrato etanólico dos capítulos de $L$. curvifolia $(2,0 \mathrm{~g})$ foi submetido ao fracionamento em coluna de permeação em gel de Sephadex LH-20, eluída com metanol. As frações de números 25-28 (101 mg) foram purificadas, utilizando uma coluna de polivinilpolipirrolidona (Sigma), eluida com metanol, fornecendo a 8-Carboximetoxi-1,6-diidroxi-3,5-dimetoxi-xantona 1 (10,0mg); as frações de números 32-36 foram purificadas nas mesmas condições que as frações 25-28, fornecendo a 8-Carboximetoxi-1,5,6-triidroxi-3-metoxi-xantona 2 (12,0mg). O extrato etanólico dos capítulos de $L$. flavescens $(2,0 \mathrm{~g})$ foi submetido ao fracionamento em coluna de Sephadex LH-20 $(100 \times 5 \mathrm{~cm})$ eluída com metanol. Foram coletadas frações de $8 \mathrm{~mL}$, que foram analisadas por cromatografia em camada delgada [gel de sílica, $n$-butanol : ácido fórmico : água (20:5:8)]. As frações de números $65-73(69,8 \mathrm{mg})$ foram reunidas e purificadas por cromatografia líquida de alta eficiência (coluna $\mathrm{C}_{18}-\mu$ Bondapak, $30 \mathrm{~cm} \times 4.6 \mathrm{~mm}$ d.i. $\times 10 \mu \mathrm{m}$ ) eluídas com metanol : água $(1: 1,1 \mathrm{~mL} / \mathrm{min})$, fornecendo a 8-Carbóximetóxi-1,3,5,6-tetraidróxi-xantona $3(4,2 \mathrm{mg}$, $\left.\mathrm{t}_{\mathrm{r}}=8 \mathrm{~min}\right)$, (Figura 1). 
<smiles>[R]c1cc(O)c2c(=O)c3c(C(C)=O)cc(O)c([R2])c3oc2c1</smiles>

\begin{tabular}{|c|c|c|}
\hline Xantonas & $\mathbf{R}_{\mathbf{1}}$ & $\mathbf{R}_{\mathbf{2}}$ \\
\hline $\mathbf{1}$ & $\mathrm{OCH}_{3}$ & $\mathrm{OCH}_{3}$ \\
\hline $\mathbf{2}$ & $\mathrm{OCH}_{3}$ & $\mathrm{OH}$ \\
\hline $\mathbf{3}$ & $\mathrm{OH}$ & $\mathrm{OH}$ \\
\hline
\end{tabular}

Figura 1. Xantonas isoladas dos extratos etanólicos dos capítulos de Leiothrix flavescens e L. curvifolia (Eriocaulaceae)

\section{Ensaios para a detecção da ação antioxidante}

Ensaio TEAC - (Trolox Equivalent Antioxidante Capacity). As substâncias puras 1, 2 e 3 foram testadas, de acordo com o método descrito por RE et al. (1999). O cátion radical ABTS ${ }^{+}$foi produzido pela reação entre $7 \mathrm{mM}$ de ABTS em $\mathrm{H}_{2} \mathrm{O}$ e $2,45 \mathrm{mM}$ de persulfato de potássio, armazenado em frasco escuro, temperatura ambiente por 12 horas. A solução de ABTS ${ }^{+}$foi então diluída com PBS $(\mathrm{pH}=7,4)$ para uma absorbância de 0,70 a $734 \mathrm{~nm}$ e mantida a $30^{\circ} \mathrm{C}$. As amostras foram diluídas em metanol, nas concentrações de 0,$3 ; 0,5 ; 1,0 ; 1,5$ e 2,0mM. A reação foi iniciada pela adição de $1 \mathrm{~mL}$ de $A B T S$ diluído em $10 \mathrm{~mL}$ de cada amostra em solução. Foram realizados três ensaios para cada amostra. A porcentagem da inibição de absorbância a $734 \mathrm{~nm}$ foi calculada para cada concentração relativa, utilizando-se metanol como branco, e foi considerada como uma função da concentração da substância ou do padrão (ácido 2-carboxílico-6-hidroxi-2,5,7,8tetrametilcromano, Trolox, Aldrich). A atividade antioxidante foi expressa em valores de TEAC, em comparação com a atividade da quercetina.

O ensaio de autoxidação utilizando o ácido linoléico foi realizado segundo o método descrito por PRATT (1992). O ácido linoléico (20 mg) e Tween 20 (200 mg) foram colocados em um erlenmeyer e, em seguida, foi adicionada uma solução contendo $2 \mathrm{mg}$ de $\beta$-caroteno em $10 \mathrm{~mL}$ de clorofórmio. Após a remoção do clorofórmio, foi adicionada uma solução de água destilada saturada com oxigênio por $30 \mathrm{~min}$. Alíquotas de $200 \mu \mathrm{L}$ de cada xantona foram dissolvidas em etanol e ajustadas para um volume final de $15 \mu \mathrm{g} / \mathrm{mL}$ de solução. Soluções sem as xantonas isoladas foram usadas como branco e uma amostra com 2,6-di-tercbutil-4-metoxifenol (BHT Aldrich) foi utilizada como substância de referência. As amostras foram submetidas à oxidação, a $50^{\circ} \mathrm{C}$, por $3 \mathrm{~h}$. A absorbância foi lida a 470 $\mathrm{nm}$ em intervalos de $1 \mathrm{e} 2 \mathrm{~h}$. A atividade antioxidante foi expressa como $\mathrm{AA}$ e calculada utilizando-se a equação: $A A \%=[1-(A o-A t) /(A o o-A o t)] \times 100 ;$ onde: Ao = Absorbância no início do experimento, com o composto teste (xantonas); At = Absorbância no tempo t com o composto teste (xantonas); Aoo = Absorbância no começo do experimento sem o composto teste (xantonas); Aot = Absorbância no tempo (t), sem o composto teste (xantonas). As substâncias são consideradas ativas quando suas AA são da mesma magnitude que a do BHT, que é a substância referência. 


\section{RESULTADOS E DISCUSSÃO}

Diversas substâncias fenólicas são importantes antioxidantes, uma vez que possuem esqueleto carbônico propício para a estabilização de radicais livres. Verificou-se que a posição e o grau de hidroxilação são fatores importantes para a atividade antioxidante dos flavonóides (LARRAURI et al., 1996). No entanto, pouco se conhece sobre a atividade antioxidante de outros compostos fenólicos derivados de plantas, como por exemplo as xantonas (MAHABUSARAKAM et al., 2000).

O método TEAC para verificar a atividade antioxidante é descrito como um ensaio de descoloração aplicável a antioxidantes lipofílicos e hidrofílicos, incluindo os flavonóides, carotenóides, hidroxinamatos e plasma (RE et al., 1999). A tabela 1 apresenta os resultados da determinação da ação antioxidante das xantonas 1, 2 e 3, isoladas de espécies de Leiothrix, obtidos pelo ensaio TEAC. Comparando-se com a quercetina (substância de referência), a atividade antioxidante relativa detectada foi de $68 \%$ para $1,55 \%$ para 2 e $40 \%$ para 3 .

Tabela 1. Atividade antioxidante das xantonas isoladas dos extratos etanólicos dos capítulos de Leiothrix flavescens e L. curvifolia (Eriocaulaceae)

\begin{tabular}{|c|c|c|c|}
\hline \multicolumn{1}{|c|}{} & ENSAIO TEAC & \multicolumn{2}{|c|}{$\begin{array}{r}\text { ENSAIO DE AUTOXIDAÇÃO } \\
\text { COM ÁCIDO LINOLÉICO }\end{array}$} \\
\hline & $(\mathrm{m} M) \pm \mathrm{DP}^{\mathrm{a}}$ & \multicolumn{2}{|c|}{ AA\% } \\
\hline Xantonas & & Após $1 \mathrm{~h}$ & Após $2 h$ \\
\hline $\mathbf{1}$ & $1,04 \pm 0,02$ & 20,81 & 22,80 \\
\hline $\mathbf{2}$ & $1,44 \pm 0,02$ & 5,82 & 11,58 \\
\hline $\mathbf{3}$ & $1,77 \pm 0,01$ & 14,91 & 17,11 \\
\hline Quercetina & $2,60 \pm 0,02$ & - & 64,1 \\
\hline BHT & - & 71,3 & - \\
\hline
\end{tabular}

${ }^{\mathrm{a}} n=3 ; \quad{ }^{\mathrm{b}}=\mathrm{BHT}=2,6$-di-tercbutil-4-metóxifenol (substância de referência)

A maior atividade apresentada pela xantona 3 poderia estar relacionada com a presença do sistema catecólico (OH-5 e OH-6) em sua estrutura. RE et al. (1999), ao estudarem os efeitos antioxidantes de vários flavonóides, verificaram que a atividade antioxidante decresce no sentido quercetina > luteolina > canferol, evidenciando que o grupo catecólico no anel B tem maior influência na atividade do que a hidroxila na posição 3 . Além disso, o maior número de hidroxilas fenólicas confere maior hidrofilicidade à xantona 3, o que também é considerado um fator de extrema importância para a atividade antioxidante (VAN DEN BERG et al., 2000).

A tabela 1 também apresenta o resultados da determinação da ação antioxidante das xantonas 1, 2 e 3, obtidos através do ensaio de autoxidação do ácido linoléico, após 1 e $2 \mathrm{~h}$. Os resultados indicam que as xantonas testadas possuem uma fraca atividade antioxidante, quando comparadas com o antioxidante de referência BHT. Após $1 \mathrm{~h}$, a atividade antioxidante calculada, 
com relação ao $\mathrm{BHT}$, foi de $29 \%$ para 1 , $8 \%$ para 2 e $21 \%$ para 3 . Após 2 h observou-se um pequeno aumento na atividade antioxidante relativa das xantonas: $36 \%$ para 1, $18 \%$ para 2 e $27 \%$ para 3. Verificou-se que atividade antioxidante relativa da xantona 1 é maior do que a das xantonas 2 e 3. Isso pode ser atribuído à presença de grupamentos metoxila nas posições C-3 e C-5, o que aumenta a lipofilicidade de 1 em relação às xantonas 2 e 3. Tendo em vista que o ácido linoléico é, também, uma substância de alta lipofilicidade, é justificável que substâncias mais lipofílicas apresentem maior atividade do que as mais hidrofílicas.

\section{CONCLUSÃO}

As plantas produzem muitos metabólitos que atuam como antioxidantes para o controle do estresse oxidativo, causado pela incidência da luz solar e oxigênio. Esses metabólitos representam uma fonte de novos compostos com atividade antioxidante, e muitos estudos já foram realizados com o objetivo de identificar substâncias antioxidantes com baixa toxicidade (SCARTEZZINI; SPERONI, 2000). A análise química de espécies da família Eriocaulaceae tem mostrado a presença de um grande número de flavonóides, naftopiranonas e xantonas. Os flavonóides já são considerados substâncias com potentes propriedades antioxidantes (SAIJA et al., 1995; VAN DEN BERG et al., 2000). Porém, modelos de outras substâncias fenólicas como xantonas ainda são pouco conhecidos. Assim, as substâncias isoladas de espécies de Eriocaulaceae podem constituir um modelo promissor para se avaliar mecanismos envolvidos na atividade antioxidante.

\section{AGRADECIMENTOS}

À FUNDUNESP e FAPESP pelo suporte financeiro à LCS e WV. Ao CNPq pela bolsa de produtividade em pesquisa concedida à WV.

\section{REFERÊNCIAS BIBLIOGRÁFICAS}

ASANO, J.; CHIBA, K.; TADA, M.; YOSHII, T. Citotoxic xanthones from Garcinia hamburyi. Phytochemistry, V.41, p.815-820, 1996.

BANERJEE, A. K., MANDAL, A., CHANDA, D., CHAKRABORTI, S. Oxidant, antioxidant and physical exercise. Molecular and Cellular Biochemistry, v.253, p.307-312, 2003.

DOKKEDAL, A.L. Estudo fitoquímico e implicações taxonômicas de Paepalanthus Kunth (Eriocaulaceae). Araraquara, 94p. Tese (Doutorado). Programa de Pós-Graduação em Química, Universidade Estadual Paulista, Instituto de Química, Araraquara, 2000.

EL-REMESSY, A. B., BEHZADIAN, M. A., ABOU-MOHAMED, G., FRANKLIN, T. CALDWELL, R. W., CALDWELL, R. B. Experimental diabetes causes breakdown of the blood-retina barrier mechanism involving tyrosine nitration and increases in expression of vascular endothelial growth factor and urokinase plasminogen activator receptor. American Journal of Pathology, v.162, p.1995-2004, 2003.

GHOSAL, S.; CHAUDHURI, R. K. Chemical constituents of Gentianaceae. Antitubercular activity of xanthones of Canscora decussata Schult. Journal of Pharmaceutical Sciences, v.64, p.888-889, 1975.

GHOSAL, S.; BISWAS, K.; CHAUDHURI, R. K. Anti Mycobacterium tuberculosis activity of naturally occurring xanthones and synthetic analogs. Journal of Pharmaceutical Sciences, v.67, p.1978, 1978.

GIULIETTI, A. M.; SCATENA, V, L.; SANO, P. T.; PARRA, L. R.; QUEIROZ, L. P.; HARLEY, R. M.; MENEZES, N. L.; YSEPPON, A. M. B.; SALATINO, A.; VILEGAS, W.; SANTOS, L. C.; RICCI, C. W.; BOMFIM, M. C. P.; MIRANDA, E. B. Multidisciplinary studies on neotropical Eriocaulaceae. In : WILSON, K. L.; MORRISON, D. A. (eds.). Monocots: systematics and evolution. Melbourne: CSIRO Pubhishing, v.1, 2000.

Rev. Bras. Farmacogn., V. 13, n. 2, jul.-dez. 2003. 
GNERRE, C.; THULL, U.; GAILLARD, P.; TESTA, B.; FERNANDEZ, E.; SILVA, F.; PINTO, M.; PINTO, M.M..; WOLFENDER, J.L.; HOSTETTMANN, K.; CRUCIANI, G. Natural and synthetic xanthones as monoamine oxidase inhibitors: biological assay and 3D-DSAR. Helvetica Chimica Acta, v.84, p552-570, 2001.

HADJIGOGOS, k. The role of free radicals in the pathogenesis of rheumatoid arthritis. Panminerva Médica, v.45, p.7-13, 2003.

HILL, R. A. Naturally occuring isocoumarins. Fortschritte der Chemie Organischer Naturstoffe, v.49, p.1-78, 1986.

JOLY A. B. Conheça a vegetação brasileira. São Paulo: EDUSP, 1970.

KUSTER, R.M.; ROCHA, L.M. Cumarinas, cromonas e xantonas. In: SIMÕES, C.M.O.; SCHENKEL, E.P.; GOSMANN, G.; MELLO, J.C.P. de; MENTZ, L.A.; PETROVICK, P.R. (Org.). Farmacognosia: da planta ao medicamento. 5.ed. Florianópolis: Editora da UFSC; Porto Alegre: Editora da Universidade, p.537-556, 2003.

LA CASA, C., VILLEGAS, I., ALACRÓN DE LASTRA, C., MOTILVA, V., CALERO, M. J.M. Evidence for protective and antioxidant properties of rutin, a natural flavone, against ethanol induced gastric lesions. Journal Ethnopharmacology, v.71, p.45-53, 2000.

LARRAURI, J. A.; GONI, I.; MARTIN-CARRON, N.; RUPEREZ, P.; SAURA-CALIXTO, F. Measurement of health promoting properties in fruit dietary fibres: Antioxidant capacity, fermentability and glucose retardation index. Journal of the Science of Food and Agriculture, v.71, p.515-519, 1996.

LIN, C. N.; LIOU, S. J.; LEE, T.H.; CHUANG, T.C.; WON, S.J. Xanthone derivatives as potencial anti-cancer dugs. Journal of Pharmacy and Pharmacology, v.48, n.5, p.539-544, 1996.

LIOU, S. S.; SHIEH, W. L.; CHENG, T. H.; WON, S. J.; LIN, C. N. Gamma pyrone compounds as potential anti-cancer drugs. Journal of Pharmacy and Pharmacology, v.45, n.9, p.191-194, 1993.

MAHABUSARAKAM, W.; PROUDFOOT, J.; TAYLOR, W.; CROFT, K. Inhibition of lipoprotein oxidation by prenylated xanthones derived from mangostin. Free Radical Research, v.33, n 5, p 643-659, 2000.

PACHUTA, R. R.; COOKS, R. G.; CASSADY, J. M.; CONG, P.; MCCLOUD, T. G.; CHANG, C. J. Antineoplasic agents from higher plants : application of tandem mass spectroscopy to xanthones from Psorospermum febrifugum. Journal of Naural Products, v.49, n.3, p.412-423, 1986.

PERES, V.; NAGEN, T. J. Naturally occurring pentaoxygenated, hexaoxygenated and dimeric xanthones: a literature survey. Química Nova, v.20, n.4, p.388-397, 1997.

PERMANA, P.A.; HO, D.K.; CASSADY, J.M.; SNAPKA, R.M. Machanism of action of the antileukemic xanhone psorospermim: DNA strand breaks, abasic sites, and protein-DNA cross-links. Cancer Res.., v.54, n.12, p.31913195, 1994.

PRATT, A. Antioxidant and cancer prevention, Washington : ACS , 1992.

RE, R.; PELLEGRINI, N.; PROTEGGENTE, A.; PANNALA, A.; YANG, M.; RICE-EVANS, C. Antioxidant activity applying an improved ABTS radical cation decolorization assay. Free Radical Biology and Medicine, v 26, n 910, p 1231-1237, 1999.

ROCHA, L.; MARTSON, A.; KAPLAN, M. A. C.; STOECKLIEVANS, H.; THULL, U.; TESTA, B.; HOSTETTMANN, $\mathrm{K}$. An antifugal gamma-pyrone and xanthones with monoamine oxidase inhibitory activity from Hypericum brasiliense. Phytochemistry, v.36, p.1381-1385, 1994.

SAIJA, A., SCALESE; M.; LANZA, M.; MARZULLO, D.; BONINA, F.; CASTELLI, F. Flavonoids as antioxidant agents: importance of their interaction with biomembrane. Free Radical Biology and Medicine, v.19, n.4, p.481486, 1995.

SANTOS, L. C.; PIACENTE, S.; PIZZA, C.; ELETTO, A. M.; DE RICARDIS, F.; VILEGAS, W. Xanthone and flavonoids from Leiothrix curvifolia and Leiothrix flavescens. Phytochemistry, v.56, p.853-856, 2001.

SCARTEZZINI, P.; SPERONI, E. Review on some plants tradicional medicine with antioxidant activity. Journal of Ethnopharmacology, v.71, p.23-43, 2000.

TAVARES, D.C.; VARANDA, E. A.; ANDRADE, F. D. P.; VILEGAS, W.; TAKAHASHI, C.S. Evaluation of the 
genotoxic potencial of the isocoumarin paeplantine in vivo and in vitro mammalian systems. Journal of Ethnopharmacology, v.68, p.115-120, 1999.

THULL, U.; KNEUBUHLER, S.; TESTA, B.; BORGES, M.F.; PINTO, M.M. Substituted xanthones as seletiveand reversible monoamine axidase A inhibitors. Pharmacological Research, v.10, n.8, p.1187-1190, 1993.

USDIN, E. Moniamine oxidase, basic and clinical frontiers. Proc Symp. Hakone Japan, Excerpta Medica, p.315-354, 1984.

VAN DEN BERG, R.; HAENEN, GRMM; VAN DEN BERG, H.; VANDER V.W.; BAST. A. The predictive value of the antioxidant capacity of structurally related flavonoids using the trolox equivalent antioxidant capacity (TEAC) assay. Food Chemistry, v.703, p.391-395, 2000.

VARANDA, E. A.; RADDI, M. S. G.; DIAS, F. L. P.; ARAUJO, M. C. S.; GIBRAN, S. C. A.; TAKAHASHI, C.S.; VILEGAS, W. Mutagenic and cytotoxic activity of an isocoumarin (paepalantine) isolated from Paepalanthus vellozioides. Teratogenesis, Carcinogemesis and Mutagenesis, v.17, p.85-95, 1997.

VILEGAS, W.; ROQUE, N.F.; SALATINO, A.; GIESBRECHT, A. M.; DAVINO, S. Isocoumarin from Paepalanthus bromelioides. Phytochemistry, v.29, p.2299-2301, 1990.

\section{*Autor para correspondência}

Profa. Dra. Lourdes Campaner dos Santos Departamento de Quìmica Orgânica Instituto de Química Universidade Estadual Paulista - UNESP Av. Prof. Francisco Degni s/n. - C.P. 355 14801-970 - Araraquara - SP e-mail: loursant@iq.unesp.br

Rev. Bras. Farmacogn., V. 13, n. 2, jul.-dez. 2003. 